\title{
Liens, corps émotionnel et langages
}

Contribution de l'approche artistique chorégraphique et fasciapulsologique à la créativité de l'expression et à la qualité de la communication

Connections, emotional body and languages: Contribution of the artistic choreographic practice and the fasciapulsologic approach towards creativity of expression and quality of communication

\section{Florence Augendre et Jacqueline Pairon}

\section{OpenEdition Journals}

\section{Édition électronique}

URL : http://journals.openedition.org/tipa/3029

DOI : 10.4000/tipa.3029

ISSN : 2264-7082

\section{Éditeur}

Laboratoire Parole et Langage

Référence électronique

Florence Augendre et Jacqueline Pairon, «Liens, corps émotionnel et langages », TIPA. Travaux interdisciplinaires sur la parole et le langage [En ligne], 35 | 2019, mis en ligne le 18 juillet 2019, consulté le 25 juillet 2019. URL : http://journals.openedition.org/tipa/3029; DOI : 10.4000/tipa.3029

Ce document a été généré automatiquement le 25 juillet 2019.

\section{(c) (†) $\odot$}

La revue TIPA. Travaux interdisciplinaires sur la parole et le langage est mise à disposition selon les termes de la licence Creative Commons Attribution - Pas d'Utilisation Commerciale - Pas de Modification 4.0 International. 


\title{
Liens, corps émotionnel et langages
}

\author{
Contribution de l'approche artistique chorégraphique et \\ fasciapulsologique à la créativité de l'expression et à la qualité de la \\ communication \\ Connections, emotional body and languages: Contribution of the artistic \\ choreographic practice and the fasciapulsologic approach towards creativity of \\ expression and quality of communication
}

Florence Augendre et Jacqueline Pairon

\section{Introduction}

1 Danseuse par nature et de formation, passionnée par toutes les formes d'expression et de création artistique, je suis également fasciapulsologue et interpellée par la force de la conjonction qui existe entre le corps et l'esprit, entre le microniveau et le macroniveau. Dans cet article, je pose l'hypothèse suivante : sous l'effet de la rapidité, l'intégration de ce qu'on est individuellement est malmenée sur un plan global et collectif et la tyrannie de l'émotion risque de s'imposer, avec une dissociation corps/ esprit. Pour moi, une attention à l'écoute intérieure avec le recentrage sur le physiologique, le respect porté aux parties fluides du corps, et un rapport au temps différent pour vivre pleinement l'instant permettent de dépasser la complexité et de s'ouvrir à une disposition plus large, plus créative qui n'est pas simplement binaire et permet une réponse responsable à différentes situations. ${ }^{5}$

2 Pour bien faire comprendre les différentes composantes de mon questionnement, je vais présenter dans un premier temps les étapes de mon cheminement empirique personnel et proposer brièvement mes principales inspirations : M. Feldenkrais et F.M. Alexander, B. Bainbridge-Cohen, C. Carini.

Ensuite, sous le prisme de mon regard d'artiste du vivant et de fasciapulsologue, j'évoquerai des éléments qui illustrent, selon moi, l'intérêt de cette conjonction des liens entre le corps et l'esprit, entre le microniveau et le macroniveau, pour le bon vécu des émotions, pour la créativité, pour la pédagogie et la pratique des langues: le corps 
émotionnel, la puissance de l'intention et l'influence de la respiration sur la voix. Nous évoquerons, en outre, de façon concrète, quelques pistes de transposition de ce regard vers le monde de l'éducation et de l'enseignement des langues.

\section{Mon parcours}

\section{1. La danseuse}

Dans le domaine de la danse, pour ma part, il y a toujours eu la possibilité de voir, sentir, trouver dans la différence des danses, des styles, des codes (ballet, jazz, claquettes, folklore traditionnel russe, danse africaine...), quelque chose de commun ou sinon quelque chose de complémentaire. Quand j'étais toute petite, j'ai fait l'expérience de points communs, car même si les sensations que chaque danse m'apportait étaient uniques, le plaisir était le même. La vibration, le sens du mouvement, les rythmes différaient ou s'articulaient selon des modalités propres à chacune, mais je ressentais toujours le sentiment d'être animée, incroyablement vivante, portée par quelque chose qui me dépassait. La possibilité de vivre toutes ces couleurs et de passer de l'un de ces éclats à l'autre magnifiait la valeur de chaque expérience dansée. Ainsi, mon corps a grandi, riche de tous ces apports culturels. Pourquoi, nous faudrait-il nous séparer, nous défaire, de ce qui nous procure tant de joie, de plaisir, de liberté ? La rupture s'est passée à l'âge de seize ans où, prenant conscience d'un certain anachronisme, j'ai décidé de mettre un terme à ma carrière classique. J'ai quitté le milieu mondain et austère de la danse classique, basée sur des formes figées que les corps des danseurs sont contraints à embrasser, et me suis tournée vers la danse contemporaine.

Une prospection dans toutes les directions possibles et non-envisagées m'amena alors à découvrir d'autres manières de vivre et créer la danse, d'être en relation symbiotique au mouvement et à sa spontanéité, "d'être dans sa propre danse ». J'ai ensuite rejoint en Belgique des troupes de danse-théatre avant-gardistes parmi lesquelles Ultima Vez de Wim Vandekeybus ${ }^{6}$ et Damaged Goods, de Meg Stuart ${ }^{7}$. Celles-ci se positionnaient dans une approche radicale et innovante par rapport aux codes du théâtre et de la danse préétablis jusqu'alors.

6 Depuis le début, je me suis demandé quelle est la nature du mouvement qui crée l'unité et quelle est sa qualité d'expression. C'est une pensée, une réflexion qui guide mes états de conscience dans le travail du corps, le développement artistique ou bien l'écoute sensible que requiert le soin. J'ai eu et continue d'avoir la grande chance de rencontrer sur mon chemin des personnes qui m'ont éclairée, ou sinon guidée. Ces rencontres faites d'échanges, de complicité et parfois de longues fidélités m'ont amenée grâce à des terreaux culturels très divers et des rapports d'âges variés à trouver suffisamment confiance et autonomie dans l'intuition qu'entre toutes ces voies diverses il y avait du lien. Cette mise en confidence n'est pas anecdotique car, grâce à ces rencontres, insensiblement, au fil de celles-ci, tout en continuant la danse, j'ai ajouté à mon parcours le travail thérapeutique qui donnait lui aussi des éléments de réponse à ma quête.

\section{2. La thérapeute}

7 Vera Orlock a été mon premier professeur en Body-Mind Centering. Cette femme exceptionnelle, danseuse, harpiste, praticienne de l'Intégration structurelle du Dr Rolf et 
de la thérapie crânio-sacrée, travaillait en tant qu'étroite collaboratrice de Bonnie Bainbridge-Cohen, la créatrice du Body-Mind Centering ${ }^{\circledR}$ (BMC ${ }^{\oplus}$ ), une approche innovante de la santé, du mouvement et du travail corporel. En effet, dès la fin des années 60 , le lien entre les notions physiologiques de la biologie du corps humain et les qualités de mouvement dansé qui leur sont relatives ont été étudiés par des danseuseschercheuses-chorégraphes, parmi lesquelles Vera Orlock. Cette approche somatique du corps-esprit, des qualités rythmiques du mouvement physique et de la voix ont donné de solides outils pour mon propre développement chorégraphique et artistique. A partir de ces prises de conscience, j'ai commencé à tisser ma propre réflexion et à la partager lors des périodes de création et durant les processus de mise en œuvre collective.

Plus tard, en parallèle de mes développements artistiques et de la pratique du BMC ${ }^{\oplus}$, je me suis intéressée à d'autres pratiques somatiques : la technique Alexander et la méthode Feldenkrais. Enfin, j'ai trouvé, auprès de Christian Carini, et par sa transmission, de convaincantes analogies avec tout ce que j'avais jusqu'alors mis en lien de façon intuitive. Ce phénomène de révélation des correspondances continue de forger ma pratique jusqu'à aujourd'hui.

Frederik Matthias Alexander pensait que les mauvaises habitudes de posture influençaient le fonctionnement du corps et de l'esprit (Paperview, 2005, p. 84). Cet acteur et pédagogue de la première moitié du XXème siècle, qui a découvert l'existence d'une certaine unité entre la tête, le cou et le dos, a mis au point une technique qui permet d'apprendre à rétablir soi-même un équilibre postural sain nécessaire au bon fonctionnement de l'organisme, en mettant en harmonie le physique et le psychique. Cette technique, qu'il a mise au point pour pallier la perte de l'usage de sa propre voix, est appelée Technique Alexander.

Moshé Feldenkrais, de nationalité russe, a étudié en France où il est devenu physicien et ingénieur. Il fut l'un des premiers en Europe à obtenir une ceinture noire de judo (en 1936) (Paperview, 2005, p. 86). Suite à une blessure au genou, et pour éviter une opération chirurgicale qui n'offrait que $50 \%$ de chance de réussite, il développa dans les années 50 une méthode de soin non conventionnelle appelée Méthode Feldenkrais.

11 Ils ont tous deux travaillé à développer une manière de trouver ou retrouver le mouvement libre de la colonne vertébrale, afin d'avoir une totale et puissante liberté de mouvement. L'un, Alexander, a tout élaboré à partir du mouvement libre de la tête. L'autre, Feldenkrais travaille exactement sur la même liberté, mais en partant de l'appui, des appuis et notamment ceux des pieds. Tous deux développent, en écho avec cette pratique du mouvement libre, une philosophie de vie et une disposition à soi.

12 De son côté, Bonnie Bainbridge-Cohen se sert du dialogue entre l'inconscient et le conscient pour permettre l'intégration de la liberté de mouvement et elle constate ceci :

Il y a un traumatisme profond à prendre conscience qu'on est au centre même de sa propre existence. (Bonnie Bainbridge-Cohen, 2016)

Ce traumatisme se manifeste dans une partie du corps chez les uns et chez d'autres dans une autre partie du corps.

14 Il est intéressant de découvrir que ces trois pratiques portent sur la même envie et nécessité d'arriver à atteindre une forme de mieux être et/ou de haute fluidité du mouvement.

15 Quant à Christian Carini, autodictate, il crée et fonde en 1979 la "Lemiscate Academy ", puis, en 1982, l'école de fasciathérapie. La Fasciapulsologie voit le jour seulement en 1988. 
Cette dernière, modèle affiné des décennies de recherche de Carini, est une thérapie manuelle qui libère par un toucher doux et profond la mémoire traumatique du corps et de l'esprit.

Selon Carini, il n'y a pas de disjonction entre toutes les strates de l'être. Mais la mémoire de certaines expériences se trouve logée dans des profondeurs accessibles uniquement par une qualité de toucher spécifique. Cette qualité requiert un état d'être, d'écoute, de reconnaissance et de présence enveloppante, dénué de l'emprise des idées ou du verbe, et qui par vagues progressives amène le patient à se détendre et à retrouver un tonus originel, proche du premier battement de la vie. Tout un phénomène de réhabilitation et d'harmonisation prend alors cours, offrant à l'individu une stabilité renforcée de son équilibre et une liberté d'expression et d'interaction avec l'environnement plus fluide.

Le corps humain se compose d'un ensemble de systèmes interactifs, le système cardio-vasculaire, digestif, endocrinien, lymphatique, musculaire, nerveux, reproducteur, respiratoire, squelettique, tégumentaire, urinaire et vasculaire dont les fonctions se coordonnent entre elles. Ces systèmes établissent entre eux un équilibre subtil, fragile, appelé homéostasie et que l'on nomme plus communément la santé.

L'homéostasie se maintient par le jeu de la nutrition, de l'élimination, de la défense et de la communication. La fasciapulsologie, s'appuyant sur le système de communication, utilise les principes actifs du sang, du système neuro-végétatif, ainsi que ceux des tissus de soutien de l'organisme. (Carini, 1995, p. 48)

Carini, à l'instar de Damasio, accorde une grande place à l'homéostasie (Damasio, 2017). Damasio, pour sa part, reconnaît dans l'homéostasie non seulement le principe de régulation d'équilibre pro-actif, mais il le considère aussi comme lieu d'élaboration de toute culture et sensibilité culturelle. (Pairon, à paraître)

Qu'énonce Damasio sur l'homéostasie? Selon lui, l'homéostasie est à la fois une régulation du vivant et une avancée vers l'avenir. Et l'immuable impératif homéostatique est l'administrateur omniprésent de la vie sous toutes ses formes. Les instruments culturels, selon lui, ont d'abord été développés pour répondre aux besoins homéostatiques des individus et des petits groupes comme les familles nucléaires et les tribus.

Le vivant nourrit un désir non réfléchi et involontaire: celui de persister et d'avancer vers l'avenir, contre vents et marées. L'homéostasie c'est « l'ensemble des processus coordonnés nécessaires à la réalisation de ce désir ». (Damasio, 2017 : interview Sciences et avenir)

Les sentiments, en leur qualité d'adjoints de l'homéostasie, sont les catalyseurs des réactions qui ont permis l'émergence des cultures humaines. Nous constatons, écrit-il, des liens entre l'organique, l'émotionnel et le socio-culturel.

Nourrie de ces approches thérapeutiques du corps, je situe le cœur de mon travail, depuis maintenant une quinzaine d'années, dans l'application de l'expérience physiologique du tissu conjonctif, appelé communément fascia et je cherche à rendre compte, par une réflexion philosophique jointe à la pratique du mouvement dansé, d'un sens non symbolique mais cohérent qui sous-tend les fondamentaux du langage (qu'il soit verbal ou infra-verbal).

21 Dans la réalité de la pratique, ma méthode commence avec quelque chose de très concret. Il s'agit de cette question : "Quelle conscience a-t-on de sa respiration ? Et dans quelle mesure est-on capable de rester conscient de son oscillation alors qu'on est à la tâche ou en mouvement?» 


\section{Les liens entre le corps et l'esprit, entre le microniveau et le macroniveau}

\section{1. Le concept de corps émotionnel}

Le corps émotionnel se révèle comme interface modulaire entre le corps physique et le corps énergétique, vu que les émotions sont l'interface entre la conscience et les phénomènes d'activité du physiologique. Le corps émotionnel est à comprendre comme interface faisant le pont, le lien, la transmission entre le corps énergétique (immatériel/ intangible) et le corps physique (substantiel/conductif). A travers cette interface, il permet de contacter nos modulations d'état les plus subtiles et il se fait aussi le reflet de notre part d'animalité, celle liée à nos plus puissants instincts de survie.

Il est observable par la pratique que les mouvements d'oscillation du corps tissulaire se répètent et aussi varient dans leur modalité d'expression, selon le degré d'intensité physiologique qui répond aux stimuli internes ou externes de l'individu, du corps-être.

Grâce à la sensation de la répétition, il nous est possible de prendre conscience de nos habitudes. Mais aussi, grâce à l'influx de mouvements énergétiques non attendus, il nous est possible de nous émanciper face à ces habitudes et de gagner confiance dans l'inconnu.

Parallèlement, grâce à l'expérience de la vitesse, il est possible de pratiquer la conjonction des sensations, de mettre en lien les informations - par association ou analogie - et de parvenir à faire des sauts entre ces différentes perceptions/expressions du temps, de l'espace en mouvement et de leurs contenus ou qualités spécifiques. L'apprentissage des langues qui nous sont étrangères me semble reposer sur la reconnaissance et l'intégration de rythmes qui sont inhabituels et extraordinaires.

C'est pourquoi, entre autres, dans ma pratique de fasciapulsologie appliquée au corps en mouvement, je parle d'un des trois fondements de notre être comme étant le 'corps émotionnel', que l'on peut retrouver dans les pratiques orientales sous la dénomination du 'corps énergétique'. À cette différence que, selon le prisme de la fasciapulsologie, en tant que pratique d'écoute et de dialogue subtil par le toucher, il est question de prendre soin de cette part de notre être qui assure de façon continue la constante transformation du tangible en intangible et de l'intangible en tangible. Cette qualité de l'être se situe à l'endroit où l'énergétique devient substantiel et le substantiel se transforme en énergétique.

Les années de recherche et d'expérimentation sur la conscience des soutiens physiologiques assurant notre mouvance et nos développements, ainsi que la pratique de certaines philosophies ancestrales du corps-esprit en mouvement, m'ont permis de vérifier que chaque organe dans notre corps a sa forme singulière, sa fonction unique, sa qualité de mouvement propre, son propre rythme et sa propre tonalité. Celles-ci peuvent être perceptibles dans la résonance particulière d'une qualité originale de la voix.

L'expertise sensible ou don du soin requiert une qualité d'écoute et de toucher permettant de sonder et reconnaitre ce qui n'est pas visible à l'œil nu, même avec l'aide des équipements médicaux de la plus haute technologie. Les informations acquises par les techniques de pointe restent des informations à interpréter et traduire, là où l'écoute et le toucher "de corps à corps ", " d'être à être ", se passe de filtre et est au plus près du 
temps réel et de la pulsation de l'instant. De cette façon, on peut reconnaître le niveau vibratoire de chaque organe pouvant s'apparenter à une sensation de couleur plus ou moins vive selon l'état de l'organe. Cette spécificité du corps organique ou émotionnel nous permet, en soin de fasciapulsologie, de reconnaître la qualité d'état physiologique du patient et si le sujet est dans un état de déséquilibre. Celle-ci permet d'identifier, si nécessaire, le degré du déséquilibre, la présence éventuelle de lésions, les organes auxquels nous pouvons apporter notre soutien, où se trouvent et comment se meuvent les dynamiques ressources.

Si chaque organe a son propre mouvement, son propre rythme et sa propre tonalité, il est aisé d'entrevoir et comprendre, que l'organisation des échanges dynamiques entre les organes est comparable à la musicalité d'une composition symphonique.

\section{2. La puissance de l'intention}

Personnellement, lorsque j'enseigne ou co-crée, j'encourage chacun, et le groupe, à sonder profondément ce qui est intentionné par chaque proposition. Alors, la manière dont chacun la reçoit sera plus déterminante et signifiante car dans cette optique, il ne s'agit pas de dupliquer des exercices ou des tâches dont on finirait par oublier l'essence et le sens. Il s'agit plutôt d'être et d'évoluer par une conscience élargie de ce qu'on est et de ce qui nous environne et de cultiver simultanément, autant que possible, une clarté d'intention. Vivre pleinement, ce qui fait sens pour notre équilibre et porter le désir de vie. Il me semble aussi important, dans un processus d'échange ou de création que chacun soit en mesure de connaitre, identifier et reconnaître ses outils et ses appuis, ainsi que la part de mouvement et de constant ré-équilibrage des dynamiques de déploiement et d'expansion de son corps. Ainsi, chacun peut découvrir le champ d'action et/ou d'expression qui lui est propre et contribuer avec autonomie, respect et consentement, au processus collectif et/ou commun. La prise en charge par chacun de son autonomie permet une véritable responsabilité, c'est-à-dire une habilité à répondre ou répondance (responsiveness). Cela favorise la créativité. Cela stimule aussi l'intelligence collective. A plus long terme, au niveau individuel comme collectif, cela renforce le ressenti de vitalité que la création et les actes créatifs induisent. Ainsi, l'estime de soi et la confiance sont vivifiées. Elles trouvent un sens et une cohérence à travers la mise à contribution et l'harmonisation du collectif. Chacun peut retrouver en soi la valeur implicite et irréductible de son aptitude à l'évolution, à la transformation et à la mise en commun. La dynamique d'émission se transforme alors, elle devient à son tour espace de réceptivité, et vice versa.

Si on reprend la notion du mouvement oscillatoire de la respiration, la fluidité d'écoute correspond à cet espace très spécial - comme un temps de latence - où le flux émis devient le flux reçu - et où inversement le flux reçu est assimilé, s'est transformé et est prêt à être extimisé (c'est-à-dire à ce qu'une part intime soit rendue publique). Cet état de latence est comparable à ces moments vécus juste avant le donner et la sensation du lâcher-prise, ainsi que juste après le recevoir et l'appréciation de reconnaissance.

En fasciapulsologie, nous comprenons (c'est-à-dire con-prendre : prendre avec) cela comme étant la manifestation des dynamiques structurelles et organiques de l'être dans l'expressivité de son corps tissulaire.

33 A cet égard, la respiration a une influence directe et incommensurable sur la voix. 


\section{3. La voix et les rythmes}

34 La qualité de notre expression vocale est directement liée à une forme d'expansion qualitative de notre respiration. Il n'y a pas de disjonction entre notre qualité respiratoire à l'instant, l'état d'équilibre de nos organes, la fluidité de communication entre eux ainsi que l'état d'isotonie (qualité intermédiaire de pression osmotique entre les flux hypotoniques et hypertoniques) de nos cellules. Pourquoi ? Parce que le geste respiratoire prend source et s'active à partir de la nécessité des cellules à recevoir la vitale dose d'oxygène, à un rythme qui lui doit rester le plus constant possible. De cette sorte, les cellules peuvent créer de l'énergie et soutenir toutes les autres fonctions vitales du corps.

La complicité avec une langue se cultive et s'entretient par le jeu des sonorités et aussi grâce au rapport de plaisir qui, à tous les niveaux, nous relie à cette langue.

Par ailleurs, "un rythme est la potentialité de toutes les articulations possibles à partir d'une pulsation " selon Pierre-Axel Izérable, musicien et compositeur d'acousmatique (technique électroacoustique de recherche et composition sonore, à partir de la fréquence physique des sons enregistrés ou créés). Je peux reconnaître cette définition dans l'analogie avec le physiologique. En effet, les cellules ont un rythme pulsatif qui a pour fonction d'assurer un équilibre stable. Parallèlement, elles s'orientent et s'organisent dans l'espace pour constituer des fibres qui elles-mêmes s'orientent et construisent les tissus. Le tissu organique se différencie en fonction de chacune des fonctions vitales qui doivent être assurées et globalement, toutes ces singularités d'échanges et de soutien se manifestent par le mouvement des flux et la résonance de leurs pulsations.

Dans l'espace interstitiel de ces échanges de flux, la possibilité d'une articulation rythmique se joue à chaque instant. La cohésion entre tous ces rythmes et la qualité harmonieuse de leurs échanges assure l'unité et l'intégrité du sujet. Cette qualité harmonieuse d'équilibre peut se ressentir lorsqu'une personne s'exprime verbalement, que ce soit dans sa langue maternelle, ou même dans une langue étrangère qu'elle maitrise peu. Nous cherchons à configurer la facilitation de ces conditions en optimisant la fluidité d'échange entre les organes.

Selon moi, le soutien des systèmes de communication physiologique est la base sur laquelle toute forme de communication peut s'appuyer, se développer et s'enrichir par l'apprentissage et grâce à l'expérience. Par analogie, si les cellules sont capables de se reproduire et de s'organiser dans l'espace et de manifester la structuration de l'espace grâce à leur capacité à se reconnaître et à se différencier ainsi qu'à incarner leur forme de conscience unique et leur cohésion de mouvement via la pulsation, on peut librement imaginer qu'au niveau des individus ces phénomènes opèrent aussi et peuvent être magnifiés.

\section{Mon questionnement dans un monde rapide et dualiste}

39 Mon questionnement porte donc sur la manière dont la nature du mouvement crée l'unité : en quoi et de quelle façon les manifestations et les qualités d'expression du mouvement assurent-elles le sens de l'unité ? Il porte ensuite sur les liens entre musicalité, rythme et corps émotionnel : comment la musicalité et les rythmes du lien 
s'appuient-ils sur les fluctuations du corps émotionnel ? Enfin, il porte sur les différentes notions d'équilibre à tous les niveaux, entre le physique et l'énergétique, entre le physiologique et le concret extérieur, entre l'intime et l'extime. Ce questionnement est d'autant plus pertinent et actuel qu'il s'inscrit dans un contexte marqué par l'accélération de la communication. Italo Calvino, déjà en 1985 lors de ses leçons américaines, pressentait l'importance de certains facteurs pour l'avenir. Il s'agit de «Lightness, Quickness, Multiplicity, Exactitude, Visibility ». Légèreté, rapidité, multiplicité, précision, visibilité. Il y a une forte demande de conjonction entre l'individuel et le collectif, entre le virtuel et le matériel, entre le tacite et l'explicite, une recherche de conjonction qui soutienne le sens de la cohérence entre tout ce que nous contactons et qui est plus au moins présent dans nos quotidiens.

Notre rapport au monde est dans un premier temps dualiste, moi et le monde, et va de pair avec nombre de dynamiques dont nous avons conscience ou auxquelles nous nous soumettons, par exemple : la faim/la satiété, le chaud/le froid, le jour/la nuit, l'intérieur/ l'extérieur, le haut/le bas, le proche/le loin, la droite/la gauche, le passé/le futur... etc. Il me semble intéressant de comprendre que ces modalités ou polarités binaires ne sont pas isolées les unes des autres. Elles concrétisent chacune une part de la base fondamentale nécessaire à notre équilibre, notre orientation et notre maintien en vie. Et ensemble, elles forment un tout qui dynamise notre élan, notre mouvement et qui nous incite à percevoir l'aspect multi-facettes de nos sensations, des perspectives qu'offre l'espace autour de nous, la qualité du contact avec les autres. Ceci n'exclut pas, dans les processus d'expérience ou d'apprentissage, les potentialités non-imaginées, parfois induites par principe vicariant. L'approche proposée ci-dessus est d'inspiration composite. Elle s'est construite à la fois à partir d'un cheminement personnel et de rencontres, de lectures et d'expériences inspirantes passées au tamis de la réflexivité.

\section{Perspectives dans le domaine de la communication/éducation et apprentissage des langues}

41 Si nous étions plus attentifs aux liens qui unissent le corps et l'esprit, le naturel et le culturel, le macroniveau et le microniveau, le ressenti et l'expression, le personnel et l'universel, notre communication ne serait-elle pas plus responsable, plus créative, plus fluide et plus souple?

42 Lorsque cet échange se manifeste et se déploie, non seulement dans un rapport en binôme mais en dépassant la dynamique dualiste du monde à travers la multiplicité des contacts potentiels ou émis, tout le panel multifacettes de l'individu peut se développer et se nuancer.

43 Ces points de rencontre sont autant de réalisations du principe conjonctif et cela assure pour un moment l'équilibre cohérent du collectif. Lorsque le collectif se reconnaît dans l'individuel, la stabilisation de l'échange peut alors profiler la naissance d'un nouveau langage.

44 Le sentiment d'appartenance ou de reconnaissance ou de possibilité de lien donne la confiance naturelle et rassurée pour que les individus s'expriment librement. La superposition des pulsations et de leurs articulations rythmiques est à la source de la 
musicalité intérieure du corps comme la prosodie est une des bases de l'intégration du langage parlé.

Par ailleurs, si nous étions capables, dans nos échanges et nos mouvements de communication, de vraiment laisser exister l'espace de latence, nos pensées et ressentis n'auraient-ils pas la possibilité de se déposer et de résonner en leurs termes propres ? Il me semble que ceci n'est pas hors de portée. Deux questions se posent dès lors, celles du pourquoi et du comment. monde sans se trahir.

- Une attention à l'écoute intérieure, un soutien à nos systèmes de communication physiologiques (respiration, attention aux parties fluides du corps, respect d'un temps de latence) sont des bases sur lesquelles toute forme de communication peut s'appuyer. En optimisant ces attentions, nous facilitons la fluidité d'échange entre les organes et améliorons l'homéostasie. Cela est déjà mis en pratique actuellement dans des ateliers pour adultes et enfants que j'anime avec des danseurs, comédiens et enseignants (Atelier du Corps en Mouvement, atelier avec enfants de 2ème maternelle - Bruxelles). Par cette approche, nous rejoignons l'intérêt porté par Damasio à l'homéostasie dans son dernier livre «L'ordre étrange des choses », avec une valorisation de l'importance prioritaire à accorder à l'équilibre interne. (Pairon, à paraître)

- Par ailleurs, avec le focus mis sur la puissance de l'intention, ce regard invite chacun à se prendre en charge et à développer une habilité à répondre de façon personnelle, à se responsabiliser (responsiveness).

52 - Dans le domaine de l'enseignement/apprentissage des langues pour assurer une meilleure écoute, une meilleure réceptivité aux sonorités et rythmes venant d'ailleurs, une souplesse, une conjonction entre le connu et l'étrange.

- L'attention à accorder à la couleur musicale et rythmique propre à chaque langue, au plaisir de jouer avec ces sons et rythmes peuvent être davantage éclairés ainsi que la mise en relation avec le paysage et la culture. Par exemple, l'âme russe pleine de fougue et de feu alliés à une profonde tendresse d'être se retrouve dans la musicalité de sa langue. 
- Cette approche non symbolique qui prend en compte le physiologique nous semble utilement compléter celle de Claire Kramsch qui, en 2007, insistait sur l'importance d'être « cunning » (habile, rusé) pour naviguer entre plusieurs langues, un peu à l'image du singe qui va de branche en branche avec souplesse. Elle soutenait l'importance de l'autonomie, nous faisons de même. Elle parlait de compétence symbolique. (Pairon, 2015) Nous mettons davantage l'accent sur l'attention au physiologique.

La compétence symbolique amenant les étudiants à "opérer entre les langues " (operate between languages, MLA 20078) ajoute une "métacouche " à tous les usages de la langue étudiés en linguistique appliquée. Cette compétence sémiotique développe une habileté à façonner (to shape) activement un environnement en multiples échelles de temps et d'espace. Ce cadre de pensée est adapté à notre époque post-moderne. Pour créer ce type de relations, il est souhaitable d'apprendre à voir par soi-même, à travers sa propre histoire incarnée et sa subjectivité ainsi qu'à travers l'histoire et la subjectivité des autres. Selon Claire Kramsch, au 21ème siècle, l'éducateur a à développer ce que Bakhtin appelle l'être « débrouillard, malin, rusé » (cunning) plutôt que seulement l'expert scientifique. La compétence symbolique, selon cette perspective écologique multilingue, est donc une habileté polyphonique. (Pairon, 2015, p. 165)

\section{Conclusion}

La relation que j'ai découverte a posteriori, c'est la force de la conjonction qui existe entre le corps et l'esprit, entre l'inné et l'acquis, entre le naturel et le culturel, le microniveau et le macroniveau. Cette force, grâce à son principe vivant et actif, se retrouve dans l'expérience de la conjonction entre le ressenti et l'expression, le personnel et l'universel. Cette recherche fait écho à d'autres démarches qui vont dans le même sens, telle celle de la comédienne Marie-Rose Meysman s'exprimant en 1996 dans le cadre de la recherche de Jacqueline Pairon.

Celui qui prend la parole devient le centre de l'attention. Comment, en même temps, rester au centre de soi-même? Il est nécessaire de rester sensible à son propre corps. Comment sortir de soi-même pour entrer dans l'univers? Le mouvement du corps est toujours l'expression exacte du mouvement de l'esprit, c'est-à-dire des mouvements de la perception, de l'émotion, de la pensée. Le geste ne devient juste que lorsque tous ces mouvements se trouvent unifiés. (Meysman, 1996, Corps et communication) ${ }^{9}$

«La démarche cognitive et sensible ensemble ». (Pairon, 1996)

Dans cet article, l'importance de la conjonction des liens a été soulignée, avec un partage pluridisciplinaire, mêlant mon regard d'artiste créatrice et mon expérience de fasciapulsologue à l'approche de Jacqueline Pairon, linguiste experte en communication ouverte aux recherches de neuroscientifiques et à celles d'artistes du vivant.

Nous avons mentionné quelques pistes pour insérer en didactique les apports de cette approche intégrative des langages qui relève de la communication, de la philosophie et donne aussi la part belle au physiologique.

Avec cette contribution, nous souhaitons faire se rejoindre, via une approche principalement somatique, les langages de l'artistique, du thérapeutique et du scientifique. Nous avons mis en lumière l'importance du sensible ainsi que l'attention à accorder aux phénomènes de régulation du vivant, pour faciliter la communication, l'apprentissage ainsi que l'expression créatrice individuelle et collective. 


\section{BIBLIOGRAPHIE}

Alexander, F. M. (2000) The Universal Constant in Living, Autriche: Mouritz.

Alexander, F. M. (2004) Constructive Conscious Control of the Individual, Autriche: Mouritz.

Bainbridge-Cohen, B. (2016) Embodying cellular consciousness through breathing and moving, Master Class, 1 \& 2 Octobre, Saue, Estonie.

Calvino, I. (2009) Six Memos for the Next Millenium, Londres: Pinguin Modern Classics.

Carini, C. (1995) Les mains du coeur, Paris: R. Laffont.

Damasio, A. (2017) L'ordre étrange des choses, Paris: Odile Jacob.

Damasio, A. (2017) Les sentiments sont les moteurs biologiques des cultures humaines, Interview pour Sciences et avenir, publié le 24 Novembre 2017 [en ligne] Disponible sur :

https://www.sciencesetavenir.fr/sante/interview-de-antonio-damasio-sur-lessentiments_118523 (consulté le 15 janvier 2019)

Feldenkrais, M. (1950) Body and Mature Behavior: A Study of Anxiety, Sex, Gravitation and Learning, New York: International Universities Press.

Feldenkrais, M. (1990) Awareness Through Movement: Health Exercises for Personal Growth, (1 ${ }^{\mathrm{èr}}$ éd. 1972, Harper \& Row), New York: Harper Collins.

Granata, P. (2011) Six memos for the next millenium [en ligne], Conférence ajoutée sur YouTube le 8 décembre 2011. Disponible sur : https://www.youtube.com/watch?v=w1PpqK2FaTg (consultée le 18 mars 2018).

Pairon, J. (2015) Présence, écoute et plurilinguisme. Vers une compétence translangagière, Le Langage et l'Homme, Vol. L, №2, p. 161-178.

Pairon, J. (à paraître) Perception des émotions, langues et cultures, in Actes de la 8ème Conférence INPRA 2018 (Intercultural Pragmatics and Communication), 8-10 juin 2018, Nicosie, Chypre.

Pairon, J. \& P. Szonyi (1996) Intérieurs d'Espaces-Temps. Sérigraphies. Composition graphique imprimée en 30 exemplaires pour le Séminaire de communication 'Parcours' à l'intention de cadres et dirigeants d'entreprise

Paperview (2005) Bien-être \& épanouissement, Bruxelles: Paperview SA

\section{NOTES}

1. Artiste chorégraphique, fasciapulsologue (certifiée IFCC Paris) et pédagogue / Choreographic artist, fasciapulsologist (certified IFCC Paris) and teacher

2. Maître de langues honoraire principal (UcLouvain) / Honorary Senior Lecturer in Languages (UcLouvain).

3. http://www.ultimavez.com/fr/ultima-vez-wim-vandekeybus

4. http://www.damagedgoods.be/

5. Cf. Animation de l'Atelier du Corps en Mouvement par Florence Augendre en 2018 (Pairon, à paraître) 
6. http://www.ultimavez.com/fr/ultima-vez-wim-vandekeybus

\author{
7. http://www.damagedgoods.be/ \\ 8. Modern Language Association \\ 9. Pairon, J. avec Pascale Szonyi (1996), Intérieurs d'Espace-Temps. Sérigraphies
}

\title{
RÉSUMÉS
}

Cet article présente le regard d'une artiste du vivant, danseuse, pédagogue et thérapeuthe en fasciapulsologie ${ }^{1}$ avec son questionnement sur la manière dont nous communiquons aujourd'hui ainsi que la transposition possible de cette approche vers le domaine de l'éducation et enseignement/apprentissage des langues. Ce regard intégratif qui semble se situer à la marge des échanges langagiers, notamment par son intérêt pour l'infra-verbal, vise à créer une meilleure synergie de communication en soi et avec les autres, via une meilleure « répondance ».

Dans une première partie, l'artiste chorégraphe présente son parcours et ses influences. Dans une deuxième partie, elle propose des éléments de son approche personnelle : le concept de corps émotionnel, la puissance de l'intention, l'influence de la respiration sur la voix et un regard original sur les rythmes. Dans une troisième partie, elle approche les différentes notions d'équilibre à tous les niveaux entre le physique et l'énergétique, entre le physiologique et le concret extérieur, entre "l'intime» et "l'extime ». Cet intérêt est pertinent dans le contexte contemporain marqué par l'accélération des communications. La quatrième et dernière partie esquisse quelques applications possibles de ces principes pour les enseignants et pour l'apprentissage des langues grâce aux éléments énoncés au sujet de l'approche somatique et de l'intelligence ancrée dans la corporéité.

La vision de l'artiste chorégraphe avec une expérience en fasciapulsologie et le regard de la linguiste experte en communication ${ }^{2}$ ouverte aux neuroscientifiques et aux artistes se rejoignent ainsi à travers une démarche qui lie le cognitif et le sensible.

This article presents the perspective of an artist working with the liveliness of the body, as a dancer, choreographer, teacher and therapist in fasciapulsology. It introduces her reflection on the way we are communicating nowadays. It reveals as well the possible transcriptions and perspectives within the realm of education. Her embodied point of view is situated at the edge of linguistic exchanges with a strong interest for the infra-verbal. It aims to create a better synergetic communication within one's self and with others, through the practice of a clearer responsiveness.

In the first part the artist-choreographer presents her itinerary and influences. The article proposes her journey as a dancer and then, her evolution as a therapist in fasciapulsology.

- the dancer : dancer by nature and by education, she has always perceived common grounds in the diverse styles of dance that she has practiced (ballet, jazz, tap-dance, traditional Russian folk dance, African dance). Her body and her experience developed, rich of these diverse cultural influences. Around the age of 16 , she decided not to continue her career in ballet, and consequently, she joined avant-garde dance-theatre companies in Belgium (Ultima Vez - Wim Vandekeybus; ${ }^{3}$ Damaged Goods - Meg Stuart ${ }^{4}$ ). One of her core questions has always concerned the nature of the movement that creates unity and its quality of expression. All these encounters 
brought her to find confidence and autonomy in her own intuition, based on the feeling that through all these paths there is connectivity.

- the therapist: Vera Orlock was her first teacher in Body-Mind Centering. This somatic approach of the body-mind, the rhythmical qualities of the physical movement and the voice, gave her skills for her own choreographic and artistic development. Later, she found interest in other somatic practices: F.M. Alexander technique, and M. Feldenkrais method. Then, she deepened her knowledge of Body-Mind Centering ${ }^{\circledR}$ with Bonnie Bainbridge-Cohen, and found with Christian Carini, self-educated and founder of Fasciapulsology, many analogies and confirmations of her intuitive practice. For him, there is no disjonction between all of the constituting layers of the being. As well as Damasio, Carini legitimates the importance of homeostasis and its balancing quality.

The second part is dedicated to elements of her personal approach: the concept of the emotional body, the inner-power of intention, the influence of breath on the voice, and her original vision on rhythms. Throughout the article she puts an emphasis on the connection between the organic and the mental, the bridge between the cellular and the universal level.

In the third part, she approaches the different notions of balance at all levels, between the physical and the energetic, between the physiologic and the external concreteness, between the 'intimate' and 'extimate'. This questioning is relevant and contemporary to a context impacted by the speed of communication. Italo Calvino (1985) was already announcing the importance of some conditioning parameters for the future (Lightness, Quickness, Multiplicity, Exactitude, Visibility). Our relation to the world, usually lived as a dual experience, is nowadays being challenged and called up to evolve in order to perceive more multifaceted aspects of our sensations, the perspectives of space around us, and our quality of contact with others.

In a fourth and last part, the article drafts some possible applications usable for teachers, teachers for teachers, and the teaching/learning of languages, in order to provide concrete tools to develop and enhance the ability of cultivating receptivity to the physiologic components. Also, integrating how the voice, the rhythms and the inner-power of intention together create pleasure and responsiveness in those applied processes.

So, this article reveals the togetherness of cognitive and sensitive approach. (Pairon, 1996, 2015). The importance of conjunction between links is underlined, with a multi-disciplinary approach, bridging the vision of a creative artist who has a fasciapulsology expertise and the approach of an expert in linguistics open to neuroscientific researches and of those engaged by artists of the liveliness.

\section{INDEX}

Mots-clés : ressenti, fluidité, écoute intérieure, oscillation, lien entre le microniveau et le macroniveau, temps de latence

Keywords : feeling, fluidity, inner listening, oscillation, bridge between the cellular and the universal level, latency-span

\section{AUTEURS}

\section{FLORENCE AUGENDRE}

Artiste chorégraphique, fasciapulsologue (certifiée IFCC Paris) et pédagogue

rubyhotcore@hotmail.com 


\section{JACQUELINE PAIRON}

Université de Louvain \& ECLE (Emotion \& Creativity in Language Education)

info@parati.be 